\title{
Can Telemetric Home Monitoring of Implantable Defibrillators Increase the Treatment's Cost-Effectiveness Ratio?
}

\author{
CH Elsner, A Dorszewski, H Kottkamp, P Schirdewahn, G Hindricks \\ Heart Center Leipzig, Leipzig, Germany
}

\begin{abstract}
Implantable defibrillators (ICDs) are the treatment of first choice concerning survived sudden cardiac death (SCD) or symptomatic ventricular tachycardias (VT).

Technical progress now offers telemetric performed interrogations and controls including information about the system status, episodes detected and treated via so called Home Monitoring (HM). Target of this work was to bundle first results and experience from single cases from a systematic clinical investigation (= Home-ICD Trial) and define a model for an economic research on the cost-effectiveness ratio of the treatment. Till today 228 patients could be included in the Trial. On the economic question a Markov-Chain Model with three abstracted types of complications and three layers of economic benefit could be set up and is shared for discussion.
\end{abstract}

\section{Introduction}

Implantable defibrillators (ICDs) are the treatment of first choice concerning survived sudden cardiac death (SCD) or symptomatic ventricular tachycardias (VT) [1] [2]. The conventional Follow-Up Scheme in this ICDTherapy is normally provided as a doctor-visit every 3 to 6 , partly 12 months. The frequency of extra visits depends on the patient's events and patients demands due to e.g. shocks / repeated episodical shocks.

During conventional physical Follow-Up normally the Collection and Analysis of Implant-Data is done, Tests with the Programming Device are performed and Pacemaker Configuration \& Patient's Medication is changed if necessary - but in a defined portion of cases it turns out, that a doctor-visit wouldn't have been necessary [3].

As shown in Figure 1 with the new Home Monitoring (HM) technology provided by Biotronik Inc. [4] it is now possible to perform telemetric longdistance interrogations and controls including information about the system status and episodes detected and treated. This continuous remote monitoring of ICD therapy can monitor the incidence of tachy- and bradycardia episodes, the therapy success rate and the actual system status. HM can provide a continuous remote monitoring of medication therapy and anti- and pro-arrhythmic effects can be performed.

Therefore with HM it seems possible to do a better allocation of resources and provide even higher quality of care with additional economic impact. One may further expect a shortened reaction time to cardiac events and especially a reduction of unnecessary routine Follow-Up.

\subsection{Objective of work}

Target of the work was to bundle first results from a systematic clinical investigation (= Home-ICD Trial) and define a raw model for a multi-layer economic research on the question of the cost-effectiveness ratio of the treatment.

With this first raw model a general draft on the treatment's cost-effectiveness ratio should be developed. The model should picture the basic setting of HM and should allow easy integration of future data from clinical investigation.

Therefore a Markov-Chain Model with three abstracted types of complications and three layers of economic benefit seemed to be feasible for this task. The model allows future simulations with different clinical data drawn from tables for each single period of investigation.

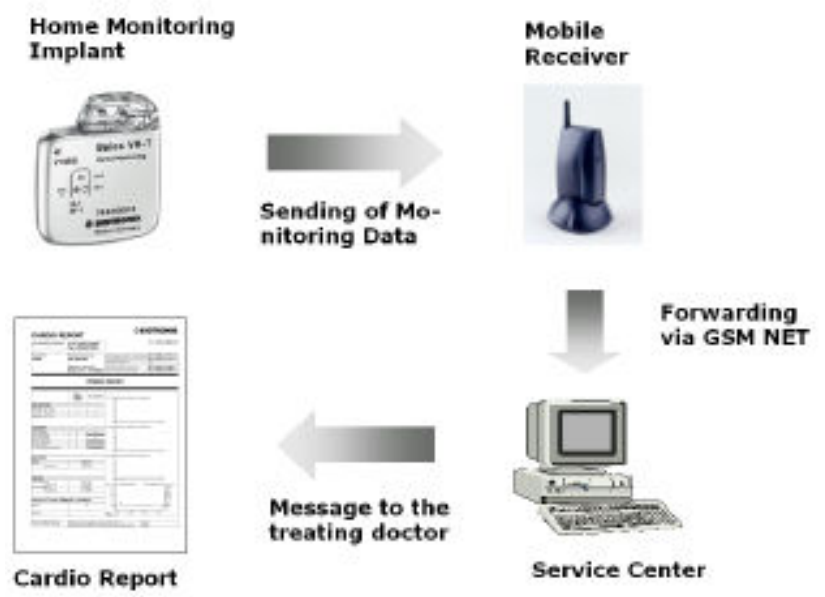

Figure 1. Workflow of Home Monitoring (HM) Technology: Pacemaker Cardio Reports over GSM NET 


\section{Methods and results}

The investigational device was a Biotronik Belos VR$\mathrm{T}$ (single chamber ICD) and DR-T (dual chamber ICD) Pacemaker. The monitored data via HM included:

- number of SVTs (DR-T), VT1, VT2 \& VF episodes

- ATPs delivered and ATPs successful

- shocks delivered, successful and aborted

- ineffective $30 \mathrm{~J}$ shocks

- Percentage of atrial \& ventricular sensing (DR-T)

- Battery voltage and status

- Atrial (DR-T) and ventricular lead impedance

HM can be performed on request, i.e. immediately, every 24 hours (performed in the Home-ICD Trial) or weekly. The event- or patient triggered messages can be turned on/off in the system setup. The reports generated can be sent over fax or over email/internet to the doctors practice. The internet report is based on a Secure-Access Webfrontend and is configurable for the doctor and provides easy workflow-oriented lists of patient reports and patient history. The standard fax-report-form being sent is seen in Figure 2. The Report tables show all transmitted data, data since last follow-Up and data since implantation. The Report graphs give overview on the episodes over time and the impedances over time.

\section{CARDIO REPORT}

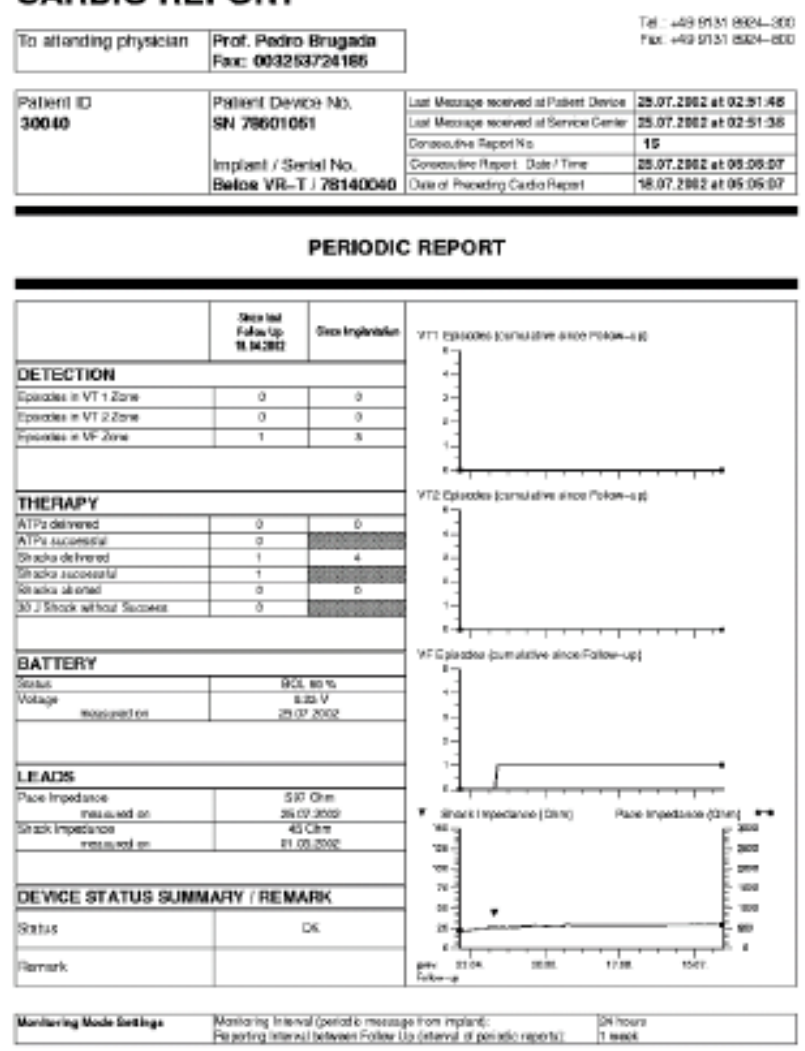

Figure 2. Picture of the standard Cardio Report sent over Fax by the system.

\subsection{First results of Home-ICD trial}

Table 1. Centers and included Patients Home-ICD Trial

\begin{tabular}{lr} 
Centers of Investigation & 24 \\
Included Patients & 228 \\
Detailed Data Patients & 196 \\
No. of Belos DR-T & 13 \\
No. of Belos VR-T & 183 \\
\hline
\end{tabular}

Table 2. Data on $n=196$ patients in Trial

\begin{tabular}{lr}
\hline Mean Age $( \pm$ SD) & $62 \pm 11$ \\
Mean LVEF $( \pm$ SD) & $40 \% \pm 16 \%$ \\
Non-compliant Pts. & 19 \\
No. of Belos DR-T & 13 \\
No. of Belos VR-T & 183 \\
\hline
\end{tabular}

As shown in Table 1 since May 2002228 patients with the given parameters (see Table 2) could be included in the trial. Indications for ICD implantation were: ventricular tachycardia (VT): $\mathrm{n}=117(60 \%)$, ventricular flutter (VF): n=55 (28\%), VT and VF: $n=8(4 \%)$ and other reasons: $\mathrm{n}=16(8 \%)$.

In 19 Patients HM couldn't be started - 14 days after release from hospital no HM message was received even after repeated requests. These Patients have been classified non-compliant and have been excluded from further investigations. After implantation implementation and testing of $\mathrm{HM}$ was performed. Routine interrogations and controls of ICD were performed 4 weeks and 3,6 and 9 months after surgery.

HM-interrogations and reports were delivered including information regarding battery status (battery), lead impedance (LI), shock impedance (SI), counts of episodes (VT/VF) and therapies delivered (ATP (overdrive stimulations) shocks).

Overall $82 \%$ percent of the reports were received successfully. Table 3 shows the data on the HM successrate.

Table 3. Data on $n=177$ patients for HM Messages

\begin{tabular}{lr}
\hline Mean Surveillance time in & $232 \pm 109$ \\
days $( \pm$ SD) & \\
"Persondays" surveillanced & 39511 \\
Messages received & 32542 \\
Percentage of surveillanced & $82 \%$ \\
days & \\
\hline
\end{tabular}

In the messages of 69 Patients (39\%) episodes could be recorded after release from the clinic. 483 VT1episodes (in 40 Patients), 67 VT2-episodes (in 13 Patients) and 249 VF-episodes (44). On the 3 Patients with most detections in each of the zones 144 detections were VT1, 52 were VT2 and 126 were VF episodes. 
In 64 Patients thearpy has been delivered by the ICD: 985 antitachycardia stimulations in 34 Patients (645 in 3 Patients), from which 389 were successfull (in 29 Patients). Mean successrate with ICD stimulations was at $70,2 \%$.

With the ICDs 332 shock-therapies were delivered in 53 Patients (155 in 3 Patients). 200 shock-therapies had to be stopped (119 in 3 Patients) and 83 have been successfull. In 14 Patients 49 ineffective shocks have been recorded (32 in 3 Patients).

\subsection{Preliminary economic results}

First results and preliminary studies on user acceptance and quality of life showed a very good acceptance and a highly significant increased feeling of security in the patients.

Preliminary Investigations on the use of a combined Fax / Internetplatform for condensed view of historical and actual patient data show good doctor acceptance and a siginificant acceleration in clinical workflow and individual diseasemanagement.

Additionally special investigations were done on the necessity of a clinical Follow-Up. Basing on the HM data the doctor had to evaluate the necessity of the clinical visit, had to perform the physical visit and then had to decide, if the visit was necessary or not.

First results show a good concordance of doctors prediction on the Follow-Up necessity from the HM Data.

Further Investigations include the detailed calculation of this data including the time needed for the different types of investigation physical vs. telemetric.

\subsection{The Markovmodel}

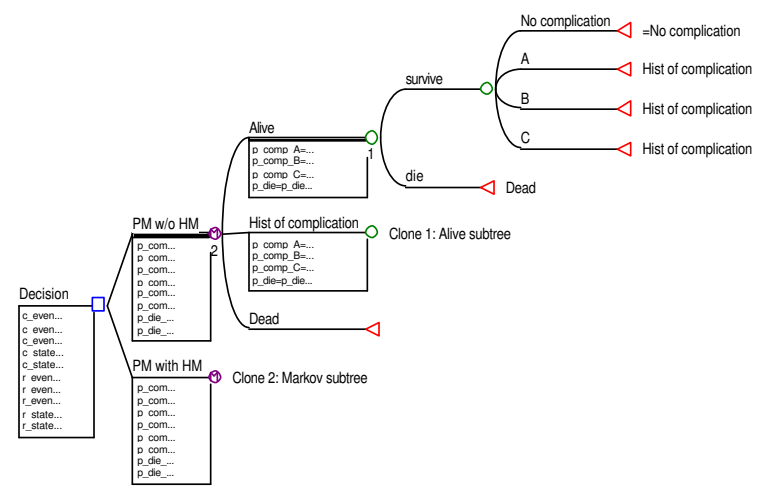

Figure 3. View of the designed Markovmodel with collapsed subtrees and collapsed formulas.

Figure 3 shows a partial view of the designed Markovmodel.

Basic principle is the (Monte Carlo) simulation of a sample number of patients running their way through the
Model for a defined number of periods. Matrices behind each node can define the costs for the virtual patients on there different layers: Real procedural Costs, Cost for Payers (=Coding) and Costdata on National Economic Scale (e.g. Costs for being absent from work or cost for travelling.)

For the different types of complications possibly occurring in the model, a simple abstraction was chosen: Type A are complications being able to be solved with HM, but lead to an unnecessary Follow-Up, Type B are complications getting worse just with HM, but being seen and/or hindered in a normal physical Follow-Up, Type C are complications with no preference for one of the two methods.

The Matrices-Based Approach is also chosen for the single possibilities to get in different states in each period. This way new or changing data can be integrated fast and easy.

\section{Discussion}

Home Monitoring improves the patient care and safety in ICD therapy by offering device status monitoring, revelation of asymptomatic episodes and continuous monitoring of device therapy.

This offers a new diagnostic tool that the patient always has with them. It seems, that this can potentially lead to a better focus on patients needing more attention than others and saving time in other populations of patients.

In our first experiences HM detected successfully repeated low-frequency VTs, inadequate therapies due to Sinustachycardia, AF and proarrhythmic effects of medication. Insufficient is the detection of electrode problems. A very positive aspect is the possibility to have control on the efficiency of therapeutic measures over HM Data.

The first investigations show good feasibility for more in depth investigations on the investigation of the costsavings in different potential patient pathways depending on the Indication for ICD-Implantation. This will hopefully lead to enough data for the developed model to run valid simulations on cost-saving aspects.

At this point of time it seems like Home Monitoring enables seamless and automatic surveillance of ICD patients and Home Monitoring enables the physician to focus on patients needing more attention than others.

Principally through limitation of scheduled and unscheduled office visits, limitation of patient's time off from work and through a potential increase of qualityadjusted lifetime the HM approach seems be good for a better Cost-Effectiveness Ratio of ICD-Treatment. Additionally the use of condensed view and reporting tools and the resulting close timeframe of monitoring allows better decisions on the necessity for measures to improve the patient's benefit from antiarrhythmic therapy and therefore potentially saves money by saving the 
patient from potential events depending on his individual indication.

The developed model allows easy step-by-step integration of data and can adapt fast to any changes and knowledge drawn from the clinical HM setting in evaluation.

The developed model seams to be feasible for further data Integration and precision of data estimations.

As the system is capable of calculation with different cost matrices for costs in real, costs for payers and costs on national economic level it seems especially capable for payer-provider negotiations in a changing healthinsurance-reimbursement environment.

\section{Acknowledgements}

Thanks to Stephané Roze and Andrew Palmer from the Center for Outcomes Research for consulting on the ongoing development of the Markovmodels.

\section{References}

[1] Prystowsky EN. Primary and secondary prevention of sudden cardiac death: the role of the implantable cardioverter defibrillator. In: Rev Cardiovasc Med (United States), Fall 2001; 2(4):197-205

[2] Mitchell LB, Roy D. Treatment approaches for patients with sustained VT/VF. In: Can J Cardiol (Canada), Jun 2000; 16 Suppl C:23C-5C

[3] Anderson M. Home Monitoring in ICD therapy: do we need it in all devices? On Venice Arrhythmias 2003 / International Workshop on Cardiac Arrhythmias, Venice October $5-82003$

[4] See URL http://www.biotronik.de/

Address for correspondence.

Christian Elsner

Thomasiusstr. 9

04109 Leipzig

Germany

Ch.Elsner@gmx.de 